\title{
AGRIS: providing access to agricultural research data exploiting open data on the web [version 1; peer review: 2
}

\section{approved]}

\author{
Fabrizio Celli ${ }^{1}$, Thembani Malapela ${ }^{1}$, Karna Wegner ${ }^{1}$, Imma Subirats ${ }^{1}$, \\ Elena Kokoliou², Johannes Keizer ${ }^{1}$
}

${ }^{1}$ Food and Agriculture Organization of the United Nations, Viale delle Terme di Caracalla, Rome, 00153, Italy

${ }^{2}$ Agro-Know, Vrilissia, 152 36, Greece

\section{V1 First published: 08 May 2015, 4:110 \\ https://doi.org/10.12688/f1000research.6354.1}

Latest published: 08 May 2015, 4:110

https://doi.org/10.12688/f1000research.6354.1

\begin{abstract}
AGRIS is the International System for Agricultural Science and Technology. It is supported by a large community of data providers, partners and users. AGRIS is a database that aggregates bibliographic data, and through this core data, related content across online information systems is retrieved by taking advantage of Semantic Web capabilities. AGRIS is a global public good and its vision is to be a responsive service to its user needs by facilitating contributions and feedback regarding the AGRIS core knowledgebase, AGRIS's future and its continuous development. Periodic AGRIS e-consultations, partner meetings and user feedback are assimilated to the development of the AGRIS application and content coverage. This paper outlines the current AGRIS technical set-up, its network of partners, data providers and users as well as how AGRIS's responsiveness to clients' needs inspires the continuous technical development of the application. The paper concludes by providing a use case of how the AGRIS stakeholder input and the subsequent AGRIS e-consultation results influence the development of the AGRIS application, knowledgebase and service delivery.
\end{abstract}

Keywords

Semantic Web, Linked Open Data, AGRIS community, agriculture, agricultural data

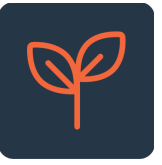

This article is included in the Agriculture, Food and Nutrition gateway.

\section{Open Peer Review}

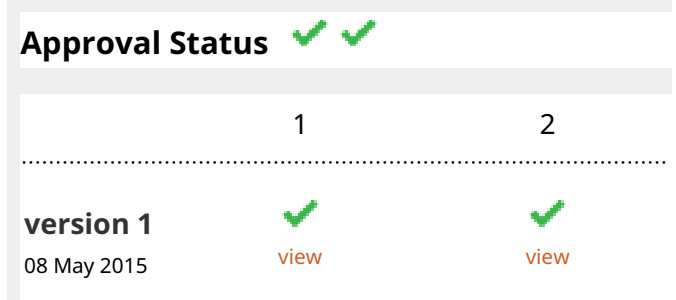

1. Laura Privalle, Bayer CropScience LP R\&D,

Morrisville, USA

2. Robert Paul Davey (ID), The Genome Analysis

Centre, Norwich, UK

Any reports and responses or comments on the article can be found at the end of the article. 
This article is included in the Data: Use and

Reuse collection.

Corresponding author: Thembani Malapela (thembani.malapela@fao.org)

Competing interests: No competing interests were declared.

Grant information: The work described in this article was partly funded by the EC project "agInfra: A data infrastructure to support agricultural scientific Communities", Grant agreement no: 283770.

Copyright: (c) 2015 Celli F et al. This is an open access article distributed under the terms of the Creative Commons Attribution License, which permits unrestricted use, distribution, and reproduction in any medium, provided the original work is properly cited. The author(s) is/are employees of the US Government and therefore domestic copyright protection in USA does not apply to this work. The work may be protected under the copyright laws of other jurisdictions when used in those jurisdictions.

How to cite this article: Celli F, Malapela T, Wegner $\mathrm{K}$ et al. AGRIS: providing access to agricultural research data exploiting open data on the web [version 1; peer review: 2 approved] F1000Research 2015, 4:110 https://doi.org/10.12688/f1000research.6354.1

First published: 08 May 2015, 4:110 https://doi.org/10.12688/f1000research.6354.1 


\subsection{Introduction}

In the last decade Semantic Web technologies have introduced changes into the way structured data is published, shared and consumed on the Web. The Web has become a powerful bedrock where emerging online applications use it as an infrastructure to exchange, query and link semantically related data and information ${ }^{1}$. In order to take advantage of the prowess of the emerging Web, many repositories have adopted linked data principles making the vision of a semantic Web of data a reality ${ }^{2}$. Overtime, two important roles of linked open data (LOD) have emerged: consuming and publishing data, thereby facilitating innovation and wider knowledge creation and sharing ${ }^{3}$. The principle of linked data has been extensively described in publications and books ${ }^{4,5}$. There are still challenges faced in browsing, analyzing, reusing and consuming linked data by the research community, Semantic Web community and policy makers. The major fallacy ${ }^{1}$ of these emerging technologies is that they assume that connectivity to data repositories and entity resolution services are always online and available.

In the agricultural domain, the Agricultural Information Management Standards (AIMS) Team of the Food and Agriculture Organization of the United Nations (FAO) has taken advantage of the possibilities of LOD in making agricultural data, information and knowledge accessible. Often-cited examples include the publication of AGROVOC (http://aims.fao.org/vest-registry/vocabularies/ agrovoc-multilingual-agricultural-thesaurus) as a linked data set $^{6}$, and the AGRIS database and application ${ }^{7}$. AGRIS (the International System for Agricultural Science and Technology) is an initiative that was set up in 1974 by the FAO to make agricultural research information discoverable and globally available. Since then AGRIS has been collecting from more than 150 data providers located in more than 65 countries. AGRIS collects and disseminates bibliographic information on scholarly and scientific publications in agriculture and related subjects.

AGRIS today is a 'global public good' ${ }^{8}$, built and maintained by a big community of data providers, partners and users. This is based on two overarching principles. Firstly, that AGRIS grants complete core access to data where users are allowed to download and use the content subject to an acceptable use policy (http://agris.fao. org/content/acceptable-use-policy). Secondly, users are invited to give ideas on the development of AGRIS and its vision through e-consultations, stakeholder meetings, user surveys and feedback. This paper will briefly overview the recent developments in AGRIS and outline the latest technical implementations. The objective of this paper is to show the responsiveness of AGRIS to the community (clients') needs and review the steps leading to the technical development and future direction of AGRIS.

\subsection{The AGRIS mashup}

Since December 2013, AGRIS has exposed its database as LOD, defining uniform resource identifiers (URIs) for bibliographic publications and allowing anyone to reuse the database also through a SPARQL endpoint. After an initial period where LOD opportunities were tested in the OpenAGRIS system ${ }^{9}$, the AGRIS team decided to adopt LOD standards into the deployed system. The goal was to take advantage of the latent knowledge available in the AGRIS data, in order to automatically discover and display related and relevant information from the Internet. AGRIS seeks to become the prime information service for agricultural research, where domain experts, agricultural extentionists, students, researchers, librarians/ information managers and decision makers can discover needed information with precision and recall it in a short response time. When the user is searching for a publication, the AGRIS system is able to enrich the user's query by displaying a mashup page with results of related information available on the same topic. To achieve this, AGRIS adopted a dual approach that allows users to access agricultural information through:

- Bibliographic metadata in the domain of agricultural science and technology are stored in a central database, currently storing nearly 8 million bibliographic references of scholarly and scientific publications.

- Other types of information (distribution maps, passport data, pictures, other bibliography, etc.) that are interlinked to the AGRIS central database.

Two things are crucial to build a useful mashup page: the selection of the data sources and the precision of the automatic extracted resources. Precision in this context means the relevance of displayed resources ${ }^{10}$ must be of the same subject coverage as that of the article selected by the user. In AGRIS this is possible through AGROVOC $^{6}$, which is a Simple Knowledge Organization System (SKOS) concept scheme used to index the AGRIS database. AGROVOC brings additional value as a thesaurus consisting of more than 32,000 concepts and is available in 21 languages, covering all areas of interest to the AGRIS database. Therefore, AGROVOC is the backbone of the resource discovery process where AGRIS records (which are indexed with AGROVOC concepts) are used to query external Web services (e.g. by scientific names) and SPARQL endpoints by using AGROVOC URIs or alignments with other thesauri related to agriculture. External data sources are identified based on the content, the relevancy to the AGRIS domain, and after evaluating, the information provider ${ }^{11}$.

Figure 1 shows a mashup page which displays an AGRIS record selected by the user with some AGROVOC descriptors and URIs (left). Once AGRIS loads the mashup page, it reads the list of AGROVOC URIs available within the AGRIS record, and run asynchronous queries to external Web services and SPARQL endpoints to get information related to the content of the selected AGRIS record. In the screenshot below for the AGROVOC concept "Oryza sativa", AGRIS displays a distribution map from GBIF (http://www. gbif.org; the Global Biodiversity Information Facility), as well as some germplasm collecting missions from Bioversity International (http://www.bioversityinternational.org/). AGRIS pulls and visualizes data from World Bank, CGRIS germplasm database, and International Food Policy Research Institute (IFPRI). A full listing of external data sources AGRIS pulls from is available on AGRIS website (http://agris.fao.org/content/how-it-works).

\subsection{AGRIS recent developments}

AGRIS is constantly evolving to provide its users with new valuable services and many new different sources of information to be explored. The development of AGRIS, both on the service and data sides, is mainly driven by AGRIS users, who can provide feedback, 


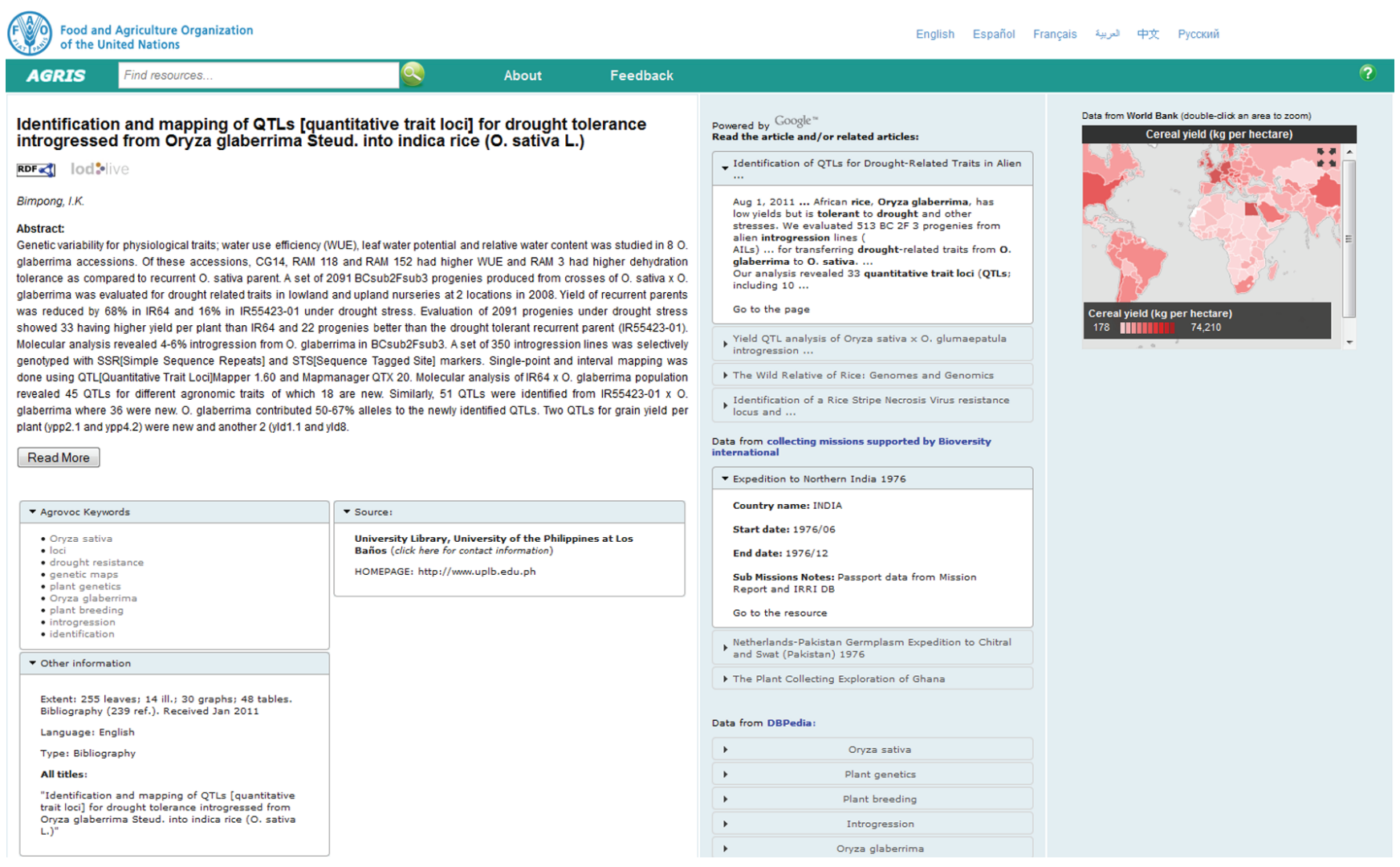

Figure 1. AGRIS mashup screenshot showing AGRIS bibliographic records pulling and visualising linked resources from external data sources.

ideas and needs in different ways: using the "feedback" form available in the AGRIS Web site; responding to periodic surveys designed by the AGRIS team; sending emails to the AGRIS Team or joining online events like AIMS Webinars [http://aims.fao.org/capacitydevelopment/webinars] or AGRIS e-consultations. All this feedback is collected and analyzed to define priorities and provide new services to the community. For instance, after the adoption of a linked data infrastructure (when AGRIS and OpenAGRIS were merged at the beginning of 2014), the AGRIS team prepared an online survey and Webinars to collect feedback about the new AGRIS Web application. Two main activities were considered as top priorities to improve the service:

1. Inclusion of all the available bibliographic metadata in the AGRIS mashup page;

2. Multilingual search with the possibility to get results in several languages when searching with keywords in a specific language.

The first activity was carried out because, even though the AGRIS Web site mashes-up many sources of information to provide its users with a good browsing experience, for many AGRIS users access to the complete bibliographic metadata set is a valuable piece of information. Thus, the mashup view was extended to include all bibliographic metadata available, and advanced search functionality (namely, "classical view") was re-introduced to allow filtering results according to specific metadata elements.

In the second activity, the multilingual search, the objective was to allow users to query the AGRIS database in their own native language, as well as retrieving results in different languages. This is exemplified by the following use case:

Xian is a Chinese researcher and he wants to discover some knowledge from the AGRIS database. He wants to know something more about "rice" and recent research activities surrounding it, but he prefers to query the database using his own native language. So he starts querying AGRIS using the keyword “稻米”. The AGRIS system discovers only 14 documents: they are not enough to add additional filters and they refer only to documents indexed with a Chinese keyword. Xian wants to access the international literature, so he also wants English articles. On the right side of the AGRIS interface, Xian enables the multilingual search and clicks on "GO": 150,000 results! Maybe now Xian has too many articles to examine, but he can use other keywords to restrict the number of the results... 


\begin{tabular}{|l|}
\hline Agrovoc Keywords \\
- veterinary hygiene \\
- disease surveys \\
- infectious diseases \\
- salmon \\
- pests of animals \\
- pathogenesis \\
- anuaculture \\
- production \\
- veterinary sciences \\
- Haematopoietic necrosis virus \\
- Japan \\
- trout \\
- viruses \\
- viroses \\
\hline Othanagement \\
"Surveillance of salmonid viruses \\
especially targeting infectious salmon \\
anemia virus in Japan" \\
Lolume: 44 \\
\hline
\end{tabular}

Fish Pathology (Journal)

FREQUENCY: Quarterly

START DATE: 1966

Agris articles from the same journal:

- Plaque assay of Oncorhynchus masou virus (OMV)

- A disease found in sea-cultured coho salmon (Oncorhynchus kisutch)

- A histopathological study of Pseudomonas fluorescens infection in tilapia

- A histopathological study on ichtyobodosis of the carp [Cyprinus carpio]

- Source:

The Agriculture, Forestry and Fisheries Research Information Technology Center (click here for contact information)

HOMEPAGE: http://www.affrc.go.jp/en/

Figure 2. Additional bibliographic metadata available in the mashup page.

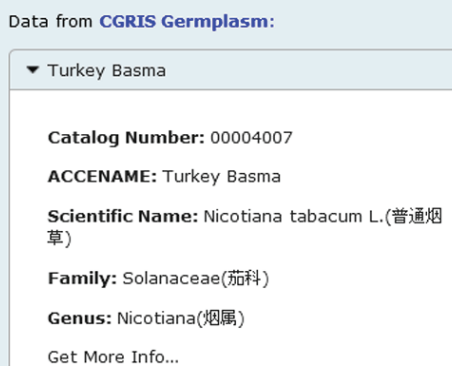

\section{发芽粘米白米黑米软罐头米饭的研制 Development of Soft Package Rice Product Made of Germinated Brown Rice,Black Rice and Rice}

到欣，当军霞

Keywords :

发芽精米; 黑米; 米的比例; 软罐头米饭

germinated brown rice;black rice;proportion of three rice;soft package rice product

\begin{abstract}
:
以发芽粗米黑米白米为原料开发研制比白米饭更具营养价值及食用方面性的软罐头米饭。通过研究3种米的比 例、发芽權米和黑米的预煮时间、蒸煮水的添加比例以及高温杀菌时间等单因素及多因素组合对软罐头米饭组 织状态、感及糊化度的影响, 确定发芽糙米白米黑米的比例为 $2: 2: 1$, 把发芽料米和黑米均分别预者 $20 \mathrm{~min}$ 和 $15 \mathrm{~min}$,蒸煮水的添加比例为 $1: 1.4$, 再和白米一起蒸者 $15 \mathrm{~min}, 121^{\circ} \mathrm{C}$ 高温杀菌 $30 \mathrm{~min}$ 可得到呈淡紫色、有光泽、

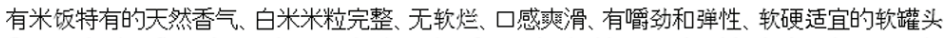
Convenient soft package rice product made of germinated brown rice, black rice, rice was developed in this paper. The proportion of the three rice,the pre-boiling time of germinated brown rice and black rice,the ratio of boiling water to whole rice,time of $h$

Citation : 食品研究与开发, 2011, (09) p. 137-141
\end{abstract}

Figure 3. The CGRIS widget showing a record integrated from the Chinese Agricultural Scitech Documents Database. This allows users to tell the data source of the main bibliographic data of the AGRIS article. 
The multilingual search is very important to facilitate access to literature in different languages: a future improvement of this feature will be offering the possibility of selecting sub-sets of languages to be included in the output of a query. The implementation of this feature relies on AGROVOC and on the AGRIS-linked open data infrastructure. In fact, while AGRIS records are indexed with AGROVOC keywords in a specific language, the translation to resource description framework (RDF) makes AGROVOC URIs usable. From an AGROVOC URI there is a possibility to extract labels of a concept in all the languages available in AGROVOC: those labels can be considered as "translations" of a query term, so that they can be used to expand the user's query to include the translation of terms in different languages. To be more precise, the implementation of the multilingual search feature required two activities:

- Indexing AGROVOC URIs in Apache Solr (http://lucene. apache.org/solr)

- Implementation of a software component that expands the user's query to match results in all languages available in AGROVOC. The query expansion is transparent to the end user, who does not need to know technical details of this feature.

Another improvement was the inclusion of Chinese research content in the AGRIS database where a large amount of Chinese metadata were directly interlinked to the AGRIS database and displayed in the mashup pages. In the context of AgINFRA (http://aginfra.eu/) and the collaboration between AGRIS and the Chinese Academy of Agricultural Sciences [http://www.caas.cn/en/administration/ research_institutes/research_institutes_beijing/77772.shtml],
500,000 resources from the Chinese Agricultural Sci-tech Documents Database (CASDD) and 410,000 resources from the CGRIS germplasm database were exposed as Web services and exploited as AGRIS external data sources, relying on the AGROVOC formal alignment with the Chinese Agricultural Thesaurus (CAT). The outcome of this activity was the inclusion of a large batch of Chinese research in agriculture in the AGRIS system, together with a unique collection of all types of plant genetic resources information from China, enriching the AGRIS knowledge base.

\subsection{AGRIS data ingestion}

AGRIS is supported by a community of data providers, partners and users. AGRIS ingests bibliographic metadata provided by the community and publishes it as open data; the metadata is captured through either (i) pulling data through harvesting from clients or (ii) by data being pushed to the AGRIS from clients? AGRIS uses various tools and technologies to consume metadata from content providers and accepts any metadata records that meet the Meaning Bibliographic Metadata (M2B) standards. AGRIS's data providers come from an international audience, with users often at varying stages of technological development. Figure 4 below summarizes the AGRIS data workflow, ingestion and processing.

The resultant AGRIS content is exposed via the AGRIS Web application - which is a mashup application that allows users to query the AGRIS content, interlinking all records to external sources of information. (See Figure 1 above and section 2.0 for more details).

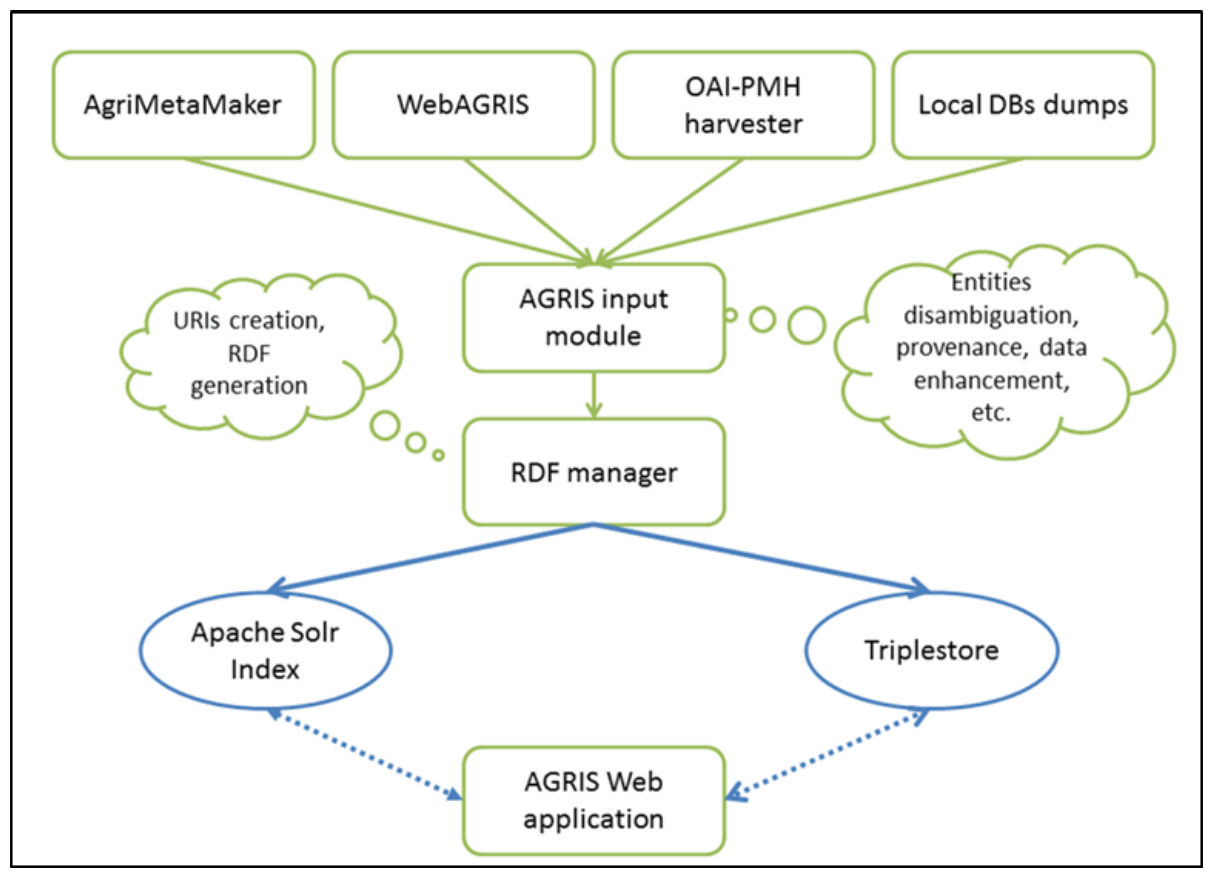

Figure 4. AGRIS dataflow and processing summarising different AGRIS data ingestion workflows and processing ${ }^{9}$. 


\subsection{AGRIS community needs}

AGRIS' vision is to be a responsive service to its global users' needs by facilitating their contribution to the AGRIS core knowledgebase, AGRIS's future and continuous development. Since 2014, FAO, Agro-Know (http://www.agroknow.gr/agroknow/) and the Agricultural Information Institute of Chinese Academy of Agricultural Sciences (http://www.caas.cn/en/administration/research_institutes/ research_institutes_beijing/77772.shtml) (CAAS) have established a collaboration for the maintenance and centralization of AGRIS data processing. The collaboration is keen to keep AGRIS a community-driven product responding to the needs of the clients. The AGRIS team has a commitment to see AGRIS visitors and data providers as clients who contribute to the continuous development of AGRIS. In pursuit of this goal, periodic AGRIS stakeholder meetings, AGRIS e-consultations in the form of online surveys and user feedback are carried out to inform the development of the AGRIS application and coverage of the knowledgebase. Four thematic areas of focus have emerged since the initial discussions: 1.) AGRIS subject coverage, 2.) geographical accessibility of the system, 3.) improvement in user interactions and multilingualism and 4.) Strengthening the infrastructural backbone of AGRIS.

In considering these thematic areas, AGRIS partners agreed to map a strategy in each respective thematic area where the resultant output will drive technical developments, new functionalities and usability features. The involvement of the AGRIS community of users and further collaboration on technical developments will be invaluable in strengthening and developing new functionalities for the AGRIS portal. Feedback received from the community of data providers, partners and users is important for the possible improvements to the AGRIS portal and the knowledgebase. Furthermore, as stated earlier AGRIS has also been involved in a number of projects with the European Commission. For example, within the SemaGrow (http://www.semagrow.eu/) project, AGRIS served as a demonstrator of a technical infrastructure based on the federation of many triple stores; relying on the two backend components Agro Tagger (http://aims.fao.org/vest-registry/tools/agrotagger) and Web Crawler, AGRIS will be able to crawl the Web and to index discovered resources with AGROVOC URIs.

The AGRIS maintenance partners sought the engagement of the broader community into the further technical developments of AGRIS in the key thematic areas outlined above. The following issues emerged in the aforementioned four key areas:

\section{AGRIS content coverage}

In terms of subject coverage, the AGRIS database collects bibliographic references in agriculture as defined by the FAO which includes nutrition, forestry, and fisheries. Since the nomenclature of AGRIS defines it as an international system for Agricultural Science and Technology, technology could also be included. The full list of subject categories can be downloaded online (http://www. fao.org/scripts/agris/c-categ.htm): they will be revised in the coming months to enable the list to cope with the increased subject coverage requirement.

In terms of content, AGRIS core data initially focused on grey literature and later came to include papers, reports and other content types. The partners felt that AGRIS backbone data should continue to be bibliographic metadata, but felt that linked data technologies should be fully exploited to allow the inclusion of other relevant content types. To further develop the coverage of AGRIS content and to prevent stagnation, the AGRIS team aims to work out a new adequate subject scope for the AGRIS knowledgebase and discover new sources of information and data in collaboration with community partners. There are possibilities of linking AGRIS with science blogs and automatically updated feeds, and of further strengthening the relationship between AGRIS and AgriFeeds (http://www.agrifeeds.org/) (for example, http://esciencenews.com and other feeds from scientific presses and universities).

Using data mining, the AGRIS database could be a way to access already existing information. In the AGRIS e-consultation users expressed their demand for more additional data like statistics, multimedia, price data, daily crops prices etc. The user survey additionally underlined the high demand for accessing full text resources. The AGRIS team has already responded with the implementation of the mashup page that allows linking to full text resources in the internet. The AGRIS Team is aware of the potential in identifying relevant content to interlink with AGRIS core data (http://aims.fao. org/activity/blog/aginfra-promotes-integration-biodiversity-information-agris). The work on providing even more full-text links and resources will be continued, with the possibility of enriching AGRIS metadata with newly discovered full-text links and of setting up a link-checking mechanism to remove broken links. There will be a need for AGRIS's authors' disambiguation and the initial option could be to use unique author identifiers, for example AGRIS intends to use the AgriVIVO's (http://aims.fao.org/vest-registry/ tools/agrivivo) scientific profiles. Another interesting activity will be the analysis of AGRIS full-text links to extract relevant information, such as a database of pictures indexed with AGROVOC, which will help enrich the content of a specific paper and to allow the re-use of pictures for personal reports or research activities, subject to copyright.

Geographical accessibility of the system and multilingualism Although AGRIS can be accessed from anywhere in the world, it has been noted that there is a lack of good performance in some regions - especially in China and East Asia. This might be due to the fact that the front-end Web application is hosted only in Rome. The AGRIS team is aware of this challenge and is trying to look for a solution to geographical accessibility by collaborating with community partners. In the meantime, the possibility of having two replicas of the database in two continents to minimize this challenge is being considered. This solution will require resources (such as a system administrator in each replica), a synchronization mechanism and a networking mechanism to geographically serve users seamlessly from different places in the world.

The user survey which registered 279 respondents who confirmed the lack of performance in some geographic regions. The overall feedback on performance was positive: around $80 \%$ of the users rated the performance of the web portal as extremely good or moderately good. Half of the users that are not satisfied with the AGRIS performance come from Asian countries. Several options to improve performance especially for countries in East Asia are 
currently being discussed and need testing. The AGRIS team must ensure that a better connection to Asia will not put other regions at a disadvantage. Recently, the multilingual search is an example of a client-demand service that has already been implemented. (see recent developments in section 3.0 above).

\section{Improvement in user interactions}

The creation of a user registration facility is one of the main goals of the AGRIS team and was demanded by the community. Both AGRIS partners and users expressed their interest for a private area that allows the creation of personal profiles and the customization of the AGRIS interface (a step towards "social AGRIS"). The implementation of functions to define the portal design and select preferred datasets in the mashup page are possible as well as the addition of social functions like comments, ratings and quoting. The AGRIS team sees potential in having AGRIS sparking debates and collaborations based on AGRIS content. Support for mobile devices (e.g. smartphones and tablets) is another possible improvement that has been demanded in the surveys and discussed with individual community partners. In the survey users regard mobile device accessibility as very important with $40 \%$ of respondents wanting AGRIS to be read on a Tablet and $24 \%$ wanting access to AGRIS on their Smartphone.

\section{Strengthening the AGRIS backbone}

The strengthening of the AGRIS backbone is important to provide a sustainable system. Currently, the AGRIS database is replicated in two types of models - (i) the AGRIS AP file system XML database and (ii) the AGRIS RDF triplestore. The AGRIS team will cease to maintain the AGRIS AP XML database and will design a new streamlined data model (most probably based on AGRIS RDF and linked open data-enabled bibliographic data (LODE-BD) (http:// aims.fao.org/lode/bd) that allows ingestion of data directly into the triple store. One of the goals is to design a more scalable and stable backend solution, as a system of load balancing of different instances of the AGRIS triplestore. In order to involve the community, the AGRIS team will have experiments as part of 'Hackathons' where participants can try different triplestore solutions simulating AGRIS queries to the database.

The above summarized feedback represents the many suggestions of the clients' needs and expectations from AGRIS. The value of the user-driven and responsive service is a core part of the AGRIS Vision and its continuous development. The move to a 'social AGRIS' will ensure that the AGRIS community of partners, data providers and users shape AGRIS service into the future. The collection of feedback from AGRIS spurs a number of potential enhancements now and in the future, with experiments made possible by the community (in the form of Hackathons). Redesign of workflows and AGRIS architecture, alongside strategic collaboration with global partners are some of the evident processes and activities within the AGRIS future vision.

\subsection{Conclusion}

AGRIS seeks to be a technological service that embraces the linked open data technologies while continuing to be a service relevant to its clients. This paper establishes that AGRIS is a global good, in that it is truly global in terms of data contribution and access, and also a public good in that it is built, maintained and responds to its community of partners, data providers, and users. AGRIS has been a bedrock for a number of semantic tools (as exhibited by the SemaGrow project) yet also provides a gateway to scientific research in Agriculture, Science and Technology. Semantic Web features have afforded AGRIS the ability to continue to be 'the' portal in Agriculture, Science and Technology for students, researchers and policy makers while at the same time constantly providing new and valuable services to the community in a dynamic and changing world.

\section{Author contributions}

FC acted as the AGRIS technical lead and decided on the conceptual content, was responsible for technical parts of this paper, contributed section 2.0 and section 3.0 and was responsible for production of all the images.

TM acted as corresponding author and coordinated inputs from all sections as well as contributing section 1.0 and part of section 5.0 and section 6.0, including the literature search. TM also fine-tuned the references and organization of the paper.

KW contributed to section 5.0, interpreted the survey results and related materials and also contributed the whole AGRIS community section.

IS provided editorial guidance and edited the paper for appropriateness regarding F1000 guidelines and correctness in discussing metadata related aspects.

EK is responsible for AGRIS data processing and contributed to section 5.0 by providing the analysis of the survey.

JK made provided guidance as to the title of the paper and direction it took, as well as taking responsibility for this content from FAO's perspective. JK also reviewed the article and cleared it for publication.

\section{Competing interests}

No competing interests were declared.

\section{Grant information}

The work described in this article was partly funded by the EC project "agInfra: A data infrastructure to support agricultural scientific Communities”, Grant agreement no: 283770. 
1. Gueret C, Boyera S, Powell M, et al: The Semantic Web for all 2014. Reference Source

2. Gayo JEL, Kontokostas D, Auer S: Multilingual Linked Data Patterns. 2012. Reference Source

3. Bauer F, Kaltenböck M: Linked open data: The essentials. A quick start guide for decision makers. 2012

Reference Source

4. Zuiderwijk A, Jeffery $K$, Janssen M: The potential of metadata for linked open data and its value for users and publishers. JeDEM. 2012; 4(2): 222-244.

Reference Source

5. Zaveri A, Rula A, Maurino A: Quality assessment methodologies for linked open data: A systematic literature review and conceptual framework. 2012.

Reference Source

6. Caracciolo C, Stellato A, Morshed A, et al.: The AGROVOC linked dataset Semantic Web. 2013; 4(3): 341-348.

Reference Source

7. Anibaldi $\mathrm{S}$, Jaques $\mathrm{Y}$, Celli F, et al:: Migrating bibliographic datasets to the Semantic Web: The AGRIS case. Semantic Web. 2015; 6(2): 113-120. Publisher Full Text

8. Rodríguez JM, Clement AJ, Farhan $\mathrm{H}$, et al:: Publishing statistical data following the linked open data principles: The web index project. In Ordonez de Pablos, P. (ed). Cases on Open-Linked Data and Semantic Web Applications. Spain: IGI. 2013; 28.

Publisher Full Text

9. Celli $F$, Jaques $\mathrm{Y}$, Anibaldi $\mathrm{S}$, et al.: Pushing, Pulling, Harvesting, Linking: Rethinking bibliographic workflows for the Semantic Web. EFITAWCCA-CIGR Conference, Turin, Italy, 24-27 June 2013. 2013. Reference Source

10. Turpin A, Scholer F: User performance versus precision measures for simple search tasks. In Proceedings of the 29th Annual international ACM SIGIR Conference on Research and Development in information Retrieval. 2006; 11-18.

Publisher Full Text

11. Jaques $Y$, Anibaldi $S$, Celli $F$, et al.: Proof and Trust in the OpenAGRIS Implementation. Proc. Int'I Conf. on Dublin Core and Metadata Applications. 2012 Implem

Reference Source 


\section{Open Peer Review}

\section{Current Peer Review Status:}

\section{Version 1}

Reviewer Report 18 August 2015

https://doi.org/10.5256/f1000research.6813.r9982

(C) 2015 Davey R. This is an open access peer review report distributed under the terms of the Creative Commons Attribution License, which permits unrestricted use, distribution, and reproduction in any medium, provided the original work is properly cited.

\section{Robert Paul Davey}

The Genome Analysis Centre, Norwich, UK

I, Robert Davey (TGAC, UK)

will sign my name to my review

will review with integrity

will treat the review as a discourse with you; in particular, I will provide constructive criticism

will be an ambassador for the practice of open science

The authors describe AGRIS, a metadata repository for an impressive cohort of bibliographic linked open data in the agricultural sciences.

Managing large-scale data generative approaches ("big"/heterogeneous data) is only one side of the coin in the modern research era. With so much data available now (and this is only going to get worse), we need systems like AGRIS to make sense of the descriptions of data to ensure that researchers can find and reuse findings more easily, and more importantly integrate them into their own work.

Whilst I too found the AGROVOC system to be a little slow and unresponsive, the AGRIS portal is relatively fast which shows the underlying power of the linked datasets, which the mashup interface represents nicely. I did attempt to use the LOD Live portion of the site, but all of the resources I tried to visualise came up with the "no resource endpoint configured". I'm not sure if this is a factor of the actual resource itself, or an issue with the query mechanism. Could the authors give some working examples?

The paper mentions the use of Lucene, but are detailed technical documents about the software implementations that power the website available? Likewise, is there any relevant source code that would be suitable for release to the community? If so, links to this information might be useful. 
The paper reads well, and gives some insight into how such a platform is built, assessed and used. The ability to search for terms in multiple languages is something that is incredibly important, and should be commended.

My only minor comments for revision would be that:

the term "mashup" might not be well understood by many. A short description or refactoring of the term might add some clarity.

I don't see the benefit of the 1.0, 2.0, etc section names. There are no subsections, so the article doesn't really need the ordered list style.

Competing Interests: No competing interests were disclosed.

I confirm that I have read this submission and believe that I have an appropriate level of expertise to confirm that it is of an acceptable scientific standard.

Reviewer Report 18 May 2015

https://doi.org/10.5256/f1000research.6813.r8691

(c) 2015 Privalle L. This is an open access peer review report distributed under the terms of the Creative Commons Attribution License, which permits unrestricted use, distribution, and reproduction in any medium, provided the original work is properly cited.

\section{Laura Privalle}

Regulatory Science Seeds and Traits Innovation Center, Bayer CropScience LP R\&D, Morrisville, NC, USA

This is an informative article on the availability of a potentially very useful software tool of which I was not previously aware. The article presents clear examples of how to use the tool and the value it brings to the user. Others will be equally interested to learn of this website.

Competing Interests: No competing interests were disclosed.

I confirm that I have read this submission and believe that I have an appropriate level of expertise to confirm that it is of an acceptable scientific standard. 
The benefits of publishing with F1000Research:

- Your article is published within days, with no editorial bias

- You can publish traditional articles, null/negative results, case reports, data notes and more

- The peer review process is transparent and collaborative

- Your article is indexed in PubMed after passing peer review

- Dedicated customer support at every stage

For pre-submission enquiries, contact research@f1000.com 